\title{
The 100 billion dollar virus
}

\author{
Ciara Harty \\ Department of Medicine, UCC
}

\section{The Scenario}

It's a Tuesday sunny afternoon; Mark is lying out in the back garden on a well-deserved break from work. He could easily stay there all afternoon, chilling out reading his book but he has a doctor's appointment at three o'clock. "It's only a check-up, maybe I could give it a miss, I do feel perfectly healthy" he thinks. Mark decided to go to the doctor's appointment convincing himself it wouldn't take long and he would feel great about himself afterwards. That evening after the appointment Mark didn't feel as good as he'd expected. The doctor had taken blood samples which were being sent away for testing with suspicion of a viral infection in his liver. This obviously worried Mark but also confused him as he felt fine. The results of the blood test show Mark is infected with a virus called Hepatitis C (HCV). It is causing damage to his liver which could result in liver failure and death.

Under his GP's recommendation, Mark visits a consultant to discuss his treatment options. He is informed that it could take up to a year to start treatment. He will undergo many medical and psychological evaluations before treatment begins. The treatment for his specific type of infection has many side effects including haemolytic anaemia, which is when your red blood cells are destroyed and you require a blood transfusion. Other side effects include depression, hair loss, stomach ulceration and vomiting. It is likely Mark will consume up to six thousand tablets during his 48-week treatment. The consultant also informs him that the success of this treatment is not guaranteed, 30-50\% of patients will fail therapy. If the treatment fails, Mark could develop resistance to further treatment meaning he will have no secondary treatment options. The cost of the treatment could be as much as $€ 50,000$. With all of this information, Mark is questioning whether he will even start this treatment. He wishes he could return to that sunny afternoon lying out in his back garden with no knowledge of the virus that has infected his liver.

\section{The Problem}

Obviously better options are needed for the 170 million people like Mark worldwide who are infected with HCV. There should be no doubt in a patient's mind whether to agree to treatment for a potentially fatal disease. Improved treatment options are badly needed. I 
am passionate about this concept and it is the focus of my $\mathrm{PhD}$ project to identify a new direction for improved therapeutics.

HCV is a virus which infects the human liver. It can lead to chronic lifelong infection, causing scarring to the liver and hindering its normal function. An infected individual is at high risk of developing cancer and requiring a liver transplant. HCV is a virus which infects 170 million people worldwide, $2.5 \%$ of the world's population. In Ireland alone it infects 30,000 to 40,000 individuals. WHO (World Health Organisation) has estimated the management cost of Hepatitis $C$ over the next decade will reach 100 billion dollars. To put this into context, the commercial giant Apple is also estimated to be worth 100 billion dollars. Should the cost of caring for HCV sufferers cost this extraordinary amount of money?

The liver is a vital organ in the human body, meaning we cannot function without it. It is the waste basket of the body, monitoring the blood and filtering out the toxins, breaking them down into non-harmful chemicals. For example, liver cells process and break down alcohol turning it into water and carbon dioxide. Blood from the digestive system passes through the liver where further absorption of compounds from our food occurs. We acquire energy from the breakdown of glucose. An important function of the liver is to absorb, store and release glucose in order to maintain the energy balance in our bodies. The liver also plays an important role in breaking down toxins acquired from the environment. Therefore, the liver is crucial to our health and disrupting its functionality, as HCV does, has severe consequences.

\section{What exactly is a virus?}

A virus is a microscopic organism which has the ability to infect and replicate in other living organisms. HCV is a sophisticated virus which infects the liver cells of humans. It consists of genetic material known as RNA and structural proteins. The RNA contains the instruction manual of how the virus acts inside the liver. The structural proteins form an envelope surrounding the delicate RNA molecule. The virus gains entry into liver cells through specific receptors on the surface of the cell. This can be compared to a locked door; the virus cannot gain entry unless it uses the correct key or receptor. Once the virus is inside the cell, the RNA is released. Now, the viral RNA redirects the cellular machinery, changing the normal processes inside the cell. This is where the problem arises. If you change the normal functions of a cell, it will not function correctly. If a cell is not functioning correctly the organ will not function correctly. If an organ is not functioning normally the human body is out of balance and our health suffers greatly. This is how HCV causes disease.

HCV can be thought of as the "fatty-virus" as it needs the fat present in the liver cell to replicate. HCV also needs a process called autophagy; this is how cells can recycle 
different components. HCV has the ability to hijack normal cellular functions such as fat metabolism and autophagy and use them to its own advantage, redirecting or changing the complicated balance of biology that allows the liver cells to function.

\section{What's the plan of action?}

My aim is to uncover new mechanisms in HCV lifecycle and find targets for novel treatment. I will focus on how HCV changes two essential functions of the cell. I am investigating how and why HCV changes how fat is used in a liver cell. While fat is normally considered unhealthy and not wanted in the body, in fact, it is needed for many functions. Lipids (another term for fat) are used for energy. They are also involved in forming internal structures and regulating hormone levels. Lipids are stored as lipid droplets inside cells, when the cell needs energy the lipid droplet is broken down. HCV increases the number of these lipid droplets which leads to a condition known as steatosis or "fattyliver". HCV changes the biology of the cell resulting in more lipid droplets being produced and fewer being broken down. In my research, I have shown an increase in the number and size of lipid droplet in liver cells infected with HCV.

I am also investigating the relationship between HCV and an immune process called autophagy, which was mentioned previously. It is a method used by cells to recycle components. Liver cells have the ability to get rid of unwanted material by breaking up that material and recycling it to be used for other purposes. Autophagy is the name of this recycling process. HCV is known to use this process to replicate. Using a technique known as immunofluorescence, I can examine the actions of HCV inside a liver cell. Immunofluorescence staining allows me to label specific proteins (viral/cellular) within the cell and visualise these proteins under a fluorescent microscope. This can be used to obtain a temporal snapshot, catching the actions of a HCV protein at a specific point in its replication cycle. An example of this can be seen in figure 1. HCV proteins are stained green, autophagy proteins are stained red. The yellow colour indicates the co-localisation of the proteins. This means that both kinds of proteins are in the same place in the cell and therefore must be interacting. By understanding exactly what the virus does inside the cell, new drugs can be developed that specifically target the virus.

My research involves investigating the life cycle of HCV with the aim of discovering what exactly the virus does inside the liver cell. I plan to identify a point of weakness in the replication of the virus, a chink in the armour of the virus. This could be used as a therapeutic target with the aim of destroying the natural progression of the virus which means no progeny virus will be produced and therefore no additional healthy cells will be infected meaning that disease progression will be halted.

New treatment options are needed for HCV patients. Mark's situation needs to be drastically improved. It is not acceptable that a treatment for a disease that affects $2.5 \%$ of the 


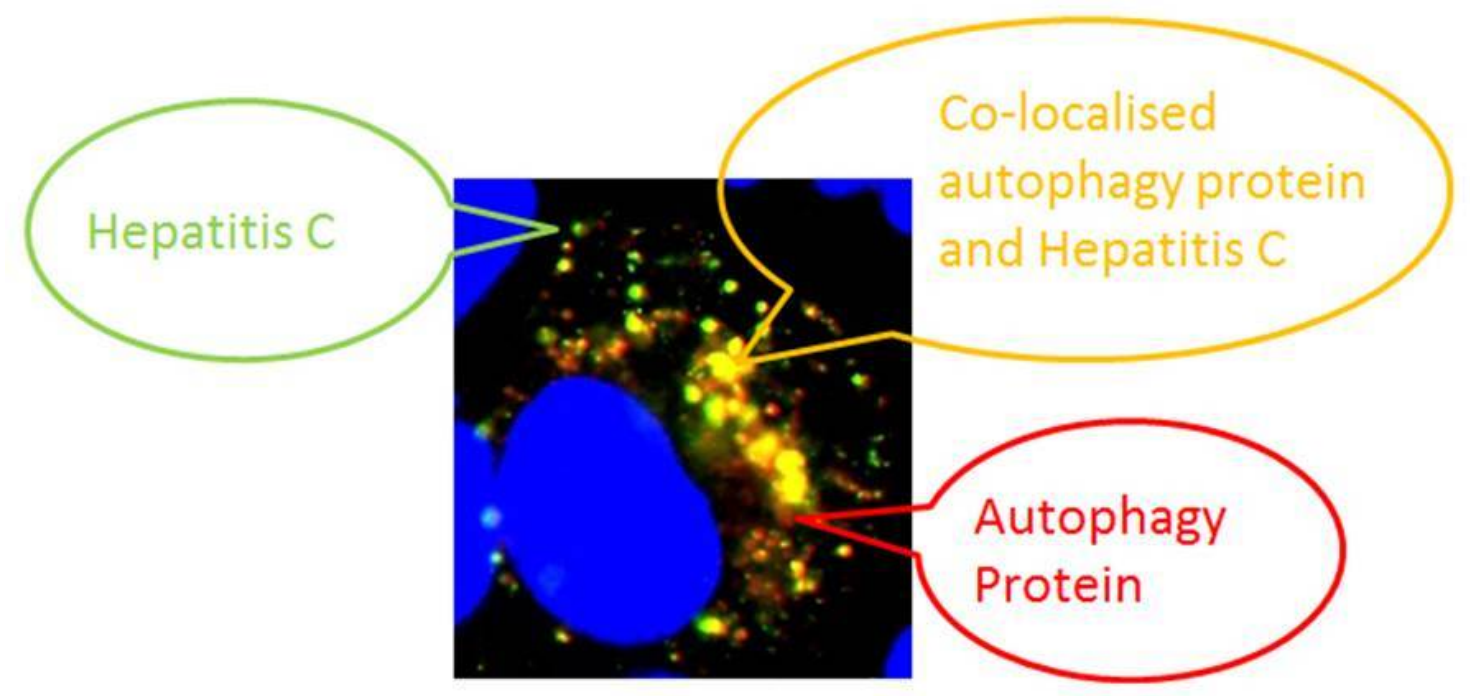

Figure 1: Liver cell infected with Hepatitis C. The image shows stained Hepatitis C proteins in green. Autophagy proteins are stained red. When the two proteins are in the same location they appear yellow. The nucleas which is the centre of the cell is stained blue. Image: Ciara Harty.

world's population has such horrendous side effects. Also, the predicted cost of 100 billion dollars for treating HCV over the next decade is unacceptable. My research aims to find a new way to treat this disease, making Mark a happier patient with a brighter future. 